\title{
Produção de biomassa micelial e enzimas lignocelulolíticas de Pleurotus spp. em meio de cultivo líquido
}

Production of mycelial biomass and lignocellulolytic enzymes of Pleurotus spp. in liquid culture medium

Producción de biomasa micelial y enzimas lignocelulolíticas de Pleurotus spp. en medio de cultivo líquido

Nelma Lopes Araújo

ORCID: https://orcid.org/0000-0002-9701-8543 Universidade Paranaense e Instituto Federal do Paraná, País E-mail: nelma.araujo@edu.unipar.br

Katielle Vieira Avelino

ORCID: https://orcid.org/0000-0001-9826-8029

Universidade Paranaense, Brasil

E-mail: katielle.avelino@edu.unipar.br

Marisangela Isabel Wietzikoski Halabura

ORCID: https://orcid.org/0000-0001-6422-911X Universidade Paranaense, Brasil

E-mail: marisangela.halabura@edu.unipar.br

Renan Alberto Marim

ORCID: https://orcid.org/0000-0003-3855-6971 Universidade Paranaense, Brasil

E-mail: renan.marim@edu.unipar.br

Adma Soraia Serea Kassem

ORCID: https://orcid.org/0000-0002-4564-1181 Universidade Paranaense, Brasil

E-mail: adma.kassem@edu.unipar.br

Thiago Teodoro Santana

ORCID: https://orcid.org/0000-0001-8523-4639

Universidade Paranaense, Brasil

E-mail: thiago.san@edu.unipar.br

Giani Andrea Linde Colauto

ORCID: https://orcid.org/0000-0003-1220-2032

Universidade Paranaense, Brasil

E-mail: gianilindecolauto@gmail.com

Nelson Barros Colauto

ORCID: https://orcid.org/0000-0003-4390-8302

Universidade Paranaense, Brasil

E-mail: nelson.c@edu.unipar.br

Juliana Silveira do Valle

ORCID: https://orcid.org/0000-0002-9463-5378

Universidade Paranaense, Brasil

E-mail: jsvalle@prof.unipar.br

\begin{abstract}
Resumo
Pleurotus spp. são fungos capazes de degradar a lignocelulose graças à excreção de enzimas com diferentes aplicações industriais. O objetivo deste estudo foi avaliar a influência dos meios de cultivo na produção de biomassa micelial e na atividade de lacase, celulases e xilanase de Pleurotus spp. Pleurotus citrinopileatus U16-23, P. djamor U16-20, U16-25 e U16-28, P. eryngii U16-30, P. ostreatus U16-22 e P. pulmonarius U16-21 foram cultivados por 12 dias a $28{ }^{\circ} \mathrm{C}$ em meio extrato de malte (EM-20 g L-1), meio definido (MD glicose $10 \mathrm{~g} \mathrm{~L}^{-1}$ e extrato de levedura $2 \mathrm{~g} \mathrm{~L}^{-}$ ${ }^{1}$ ), ou em meio bagaço de cana-de-açúcar (BC) igual ao $\mathrm{MD}$, mas substituindo glicose por bagaço $\left(50 \mathrm{~g} \mathrm{\textrm {L } ^ { - 1 }}\right)$. $\mathrm{O} \mathrm{maior}$ crescimento ocorreu no meio EM e $P$. djamor U16-25 a cepa que gerou mais biomassa $\left(7,5 \pm 0,1 \mathrm{~g} \mathrm{~L}^{-1}\right)$. A maioria das cepas apresentou maior atividade de lacase no meio MD e as atividades maiores foram de $P$. djamor U16-25 (22243 \pm $\left.745 \mathrm{U} \mathrm{L}^{-1}\right)$ e $P$. pulmonarius (20924 $\left.\pm 46 \mathrm{U} \mathrm{L}^{-1}\right)$. P. citrinopileatus e $P$. djamor U16-25 não produziram celulases, mas $P$. djamor U16-20 e $P$. eryngii produziram as maiores atividades de celulase nos meios EM e MD. Todas as cepas produziram xilanase em e as maiores atividades foram observadas no meio EM e P. djamor U16-28 (67154 \pm 1597 U
\end{abstract}


$\mathrm{L}^{-1}$ ) foi o maior produtor. Pleurotus djamor U16-25 se destacou como produtor de lacase e de xilanase livre de celulase revelando potencial para aplicação na indústria de papel e celulose.

Palavras-chave: Basidiomycota; Celulase; Cogumelo-ostra; Lacase; Meio de cultivo; Xilanase.

\begin{abstract}
Pleurotus spp. are fungi capable of degrading lignocellulose material due to the excretion of enzymes with different industrial applications. This study aimed to investigate the influence of culture media on the production of mycelial biomass and the activity of laccase, cellulases, and xylanase of Pleurotus spp. Pleurotus citrinopileatus U16-23, $P$. djamor U16-20, U16-25, and U16-28, P. eryngii U16-30, $P$. ostreatus U16-22, and $P$. pulmonarius U16-21 were grown for 12 days at $28{ }^{\circ} \mathrm{C}$ in malt extract medium (EM-20 g L-1), defined medium (MD glucose $10 \mathrm{~g} \mathrm{~L}^{-1}$ and yeast extract $\left.2 \mathrm{~g} \mathrm{~L}^{-1}\right)$, or in sugarcane bagasse (BC) medium equal to $\mathrm{MD}$, but replacing glucose with bagasse $\left(50 \mathrm{~g} \mathrm{~L}^{-1}\right)$. The greatest growth occurred in the medium EM and P. djamor U16-25 was the strain that generated the greatest biomass $\left(7.5 \pm 0.1 \mathrm{~g} \mathrm{~L}^{-1}\right)$. Most strains showed higher laccase activity in the MD medium and the greatests activities were P. djamor U16-25 (22243 $\left.\pm 745 \mathrm{U} \mathrm{L}^{-1}\right)$, and P. pulmonarius (20924 $\left.\pm 46 \mathrm{U} \mathrm{L}^{-1}\right)$. P. citrinopileatus and $P$. djamor U16-25 did not produce cellulases, but $P$. djamor U16-20 and $P$. eryngii produced the greatest cellulase activities in EM and MD media. All strains produced xylanase, and the highest activities were observed in the EM medium and $P$. djamor U16-28 (67154 $\left.\pm 1597 \mathrm{U} \mathrm{L}^{-1}\right)$ was the best producer. Pleurotus djamor U16-25 excelled as a producer of laccase and cellulase-free xylanase, revealing the potential for application in the paper and cellulose industry.
\end{abstract}

Keywords: Basidiomycota; Cellulase; Culture medium; Laccase; Oyster mushroom; Xylanase.

\title{
Resumen
}

Pleurotus spp. son hongos capaces de degradar la lignocelulosa debido a la excreción de enzimas con diferentes aplicaciones industriales. Este estudio tuvo como objetivo investigar la influencia de los medios de cultivo en la producción de biomasa micelial y la actividad de lacasa, celulasas y xilanasa de Pleurotus spp. Pleurotus citrinopileatus U16-23, $P$. djamor U16-20, U16-25 y U16-28, $P$. eryngii U16-30, $P$. ostreatus U16-22 y $P$. pulmonarius U16-21 se cultivaron durante 12 días a $28{ }^{\circ} \mathrm{C}$ en medio extracto de malta (EM-20 g L-1), medio definido (MD glucosa $10 \mathrm{~g} \mathrm{~L}^{-1}$ y extracto de levadura $2 \mathrm{~g} \mathrm{~L}^{-1}$ ), o en medio de bagazo de caña de azúcar (BC) igual a $\mathrm{MD}$, pero en sustitución de glucosa con bagazo $\left(50 \mathrm{~g} \mathrm{~L}^{-1}\right)$. El mayor crecimiento se presentó en el medio EM y P. djamor U1625 fue la cepa que generó mayor biomasa $(7.5 \pm 0.1 \mathrm{~g} \mathrm{~L}-1)$. La mayoría de las cepas mostraron mayor actividad lacasa en el medio MD y las mayores actividades fueron P. djamor U16-25 (22243 \pm 745 U L-1) y P. pulmonarius (20924 \pm $\left.46 \mathrm{U} \mathrm{L}^{-1}\right)$. $P$. citrinopileatus y $P$. djamor U16-25 no produjeron celulasas, pero $P$. djamor U16-20 y $P$. eryngii produjeron las mayores actividades de celulasa en los medios EM y MD. Todas las cepas produjeron xilanasa, y las mayores actividades se observaron en el medio EM y P. djamor U16-28 (67154 $\left.\pm 1597 \mathrm{U} \mathrm{L}^{-1}\right)$ fue el mejor productor. Pleurotus djamor U16-25 se destacó como productor de de lacasa y xilanasa libre de celulasa, revelando el potencial de aplicación en la industria del papel y la celulosa.

Palabras clave: Basidiomycota; Celulasa; Lacasa; Medios de cultivo; Seta de ostra; Xilanasa.

\section{Introdução}

Estima-se que a produção anual mundial de cogumelos comestíveis seja de 34 milhões de toneladas com um mercado global de US\$ 30 a 60 bilhões por ano (Grimm \& Wösten, 2018). As espécies de fungos produtores de cogumelos do gênero Pleurotus (Fr.) P. Kumm. (Pleurotaceae) estão em segundo lugar no mundo em produção de cogumelos e apenas na China cerca de seis milhões de toneladas de Pleurotus, principalmente $P$. ostreatus e $P$. citrinopileatus, são produzidos anualmente (Royse et al., 2017). Várias espécies do gênero apresentam importância comercial como $P$. ostreatus (cogumelo-ostra), $P$. eryngii (cogumelo-ostra-rei), P. djamor (cogumelo-ostra-rosa) e P. citrinopileatus (cogumelo-ostra-dourado) (Royse et al., 2017; Sekan et al., 2019). Apreciados mundialmente por seu aroma e sabor, cogumelos Pleurotus spp. também são conhecidos por suas propriedades nutricionais (Fernandes et al., 2015; Rampinelli et al., 2010), por produzirem compostos bioativos com atividade antioxidante (Mishra et al., 2013; Umeo et al., 2015), imunomoduladora, antitumoral, anti-inflamatória (Vetvicka et al., 2019; Yin et al., 2019), por bioacumularem metais e serem usados como alimentos funcionais (Faria et al., 2018; Scheid et al., 2020; Yokota et al., 2016).

Pleurotus spp. são espécies de fungos da podridão branca e, por isso, podem crescer em substratos lignocelulósicos devido à capacidade de degradar todos os componentes da parede celular vegetal, produzindo enzimas extracelulares para a 
degradação da lignina, celulose e hemicelulose (Knop et al., 2015; Ravindran et al., 2018). As principais enzimas desse complexo são hidrolases (celulases e hemicelulases) e ligninases (fenol oxidases e peroxidases) (Elisashvili et al., 2017). Estas classes de enzimas degradam a parede celular vegetal fornecendo ao fungo nutrientes como carbono, nitrogênio, enxofre, entre outros; fazendo com que os fungos tenham habilidade de crescer em diferentes substratos (Bellettini et al., 2019). Este complexo enzimático de fungos pode ser aplicado em diferentes indústrias como química, agrícola, alimentícia, medicinal, de energia (Barcelos et al., 2020), para degradar compostos tóxicos como corantes (Kunjadia et al., 2016), fármacos (Singh et al., 2017) e outros poluentes orgânicos (Bettin et al., 2019; Jové et al., 2016).

O complexo celulolítico envolve a ação sinérgica de enzimas como as endo-1,4- $\beta$-D-glicanases ou endoglicanases, exo-1,4- $\beta$-D-glicanases ou celobiohidrolases e 1,4- $\beta$-D-glicosidases. As endoglicanases são responsáveis pela hidrólise da celulose, expondo as extremidades da cadeia facilitando a atuação da celobiohidrolases nas extremidades criadas e liberando oligossacarídeos como as unidades de celobiose. As glicosidases degradam a celobiose liberando glicose (Várnai et al., 2010). Xilanases são responsáveis pela degradação de ligações $\beta-1,4$ da xilana vegetal, um dos principais componentes da hemicelulose (Bhardwaj et al., 2019) e as principais xilanases são a $\beta$-1,4-endoxilanase e a $\beta$-D-xilosidase (Menezes et al., 2009). As endoxilanases degradam as ligações glicosídicas da xilana de forma aleatória reduzindo a polimerização do substrato e liberando xilo-oligossacarídeos (Menezes et al., 2009), enquanto as $\beta$-xilosidases hidrolisam os xilo-oligossacarídeos (Hasmann et al., 2003). Lacases são cobre polifenol oxidases, capazes de oxidar substratos fenólicos gerando radicais livres correspondentes enquanto reduzem o oxigênio à água (Sharma et al., 2018) e acredita-se que lacases tenham papel central na despolimerização da lignina e em detoxificação degradando subprodutos tóxicos derivados da degradação da lignina (Moreno et al., 2014).

O mercado global de enzimas industriais deve atingir US\$7,0 bilhões até 2023, com crescimento anual de 4,9\% para o período 2018-2023 (BCC Research, 2018). Enzimas são preferidas como biocatalisadores em processos industriais devido ao seu elevado grau de especificidade, seletividade e parâmetros operacionais robustos (Anugraha et al., 2016). Contudo, enzimas são componentes caros e isso estimula a bioprospecção de espécies produtoras, além de pesquisa em estratégias de cultivo e produção para a obtenção de novas enzimas com características específicas variadas e que possam ser produzidas a um custo menor (Ravindran et al., 2018).

O crescimento micelial e a produção de enzimas durante o cultivo de fungos são influenciados por fatores biológicos, químicos e físicos como linhagem ou espécie de fungo, quantidade de inóculo, fontes de nutrientes, pH, temperatura, aeração, umidade e luminosidade (Ritota \& Manzi, 2019). Fontes de nutrientes fornecidos no meio de cultivo como elementos prontamente metabolizáveis ou como fontes complexas podem provocar mudanças fisiológicas que afetam a expressão e a produção de enzimas (Bellettini et al., 2019). Assim, é importante conhecer as condições básicas de cultivo em meio líquido quando o objetivo é produzir enzimas com potencial biotecnológico (Tavares et al., 2020). O objetivo deste estudo foi avaliar a influência de diferentes meios de cultivo na produção de biomassa micelial e na atividade de lacase, celulase e xilanase de Pleurotus spp.

\section{Metodologia}

\subsection{Espécies de Pleurotus e produção de inóculo}

Os ensaios foram conduzidos com diferentes cepas e espécies de Pleurotus: Pleurotus citrinopileatus Singer U16-23 cepa comercial asiática (M22502, Mycelia Mushroom Spawn Company, Bélgica); Pleurotus djamor (Rumph. ex Fr.) Boedijn U16-25 e U16-28 cepas isoladas no México (IE870, sinonímia Pleurotus djamor var. roseus do Instituto de Ecologia, 
INECOL, México); Pleurotus djamor U16-20 cepa comercial asiática (M2708, Mycelia Mushroom Spawn Company, Bélgica); Pleurotus eryngii (DC.) Quél. U16-30 cepa de origem europeia (IE689, Instituto de Ecologia, INECOL, México); Pleurotus ostreatus (Jacq.) P. Kumm. U16-22, cepa comercial europeia (M2191, Mycelia Mushroom Spawn Company, Bélgica) e Pleurotus pulmonarius (Fr.) Quél. U16-21 cepa comercial europeia (M2204, Mycelia Mushroom Spawn Company, Bélgica). Todas as cepas foram depositadas na coleção de culturas do Programa de Pós-Graduação em Biotecnologia Aplicada à Agricultura da Universidade Paranaense e criopreservadas de acordo com Zaghi Junior et al. (2020).

Os fungos foram cultivados em placas de Petri contendo ágar-extrato-de-malte (AEM, extrato de malte $20 \mathrm{~g} \mathrm{~L}^{-1} \mathrm{e}$ ágar $\left.10 \mathrm{~g} \mathrm{~L}^{-1}\right)$ a $28 \pm 1^{\circ} \mathrm{C}$ por sete dias para a produção de inóculo. Três discos de $6 \mathrm{~mm}$ de diâmetro contendo micélio foram utilizados para inocular os meios de cultivo líquidos.

\subsection{Avaliação do crescimento micelial e da produção de enzimas em meio líquido}

As linhagens foram cultivadas em três meios de cultivo líquidos diferentes: meio extrato de malte (EM), meio definido (MD) e meio com bagaço de cana-de-açúcar (BC). O meio EM continha extrato de malte $20 \mathrm{~g} \mathrm{~L}^{-1}$; o meio MD foi composto por glicose $10 \mathrm{~g} \mathrm{~L}^{-1}$, extrato de levedura $2 \mathrm{~g} \mathrm{~L}^{-1}, \mathrm{KH}_{2} \mathrm{PO}_{4} 1,5 \mathrm{~g} \mathrm{~L}^{-1}, \mathrm{MgSO}_{4} 0,5 \mathrm{~g} \mathrm{~L}^{-1}, \mathrm{CaCl}_{2} 0,1 \mathrm{~g} \mathrm{~L}^{-1}, \mathrm{FeSO}_{4} 0,036 \mathrm{~g}$ $\mathrm{L}^{-1}, \mathrm{ZnSO}_{4}$ 0,03 g L-1 (Valle et al., 2014); e o meio BC tinha composição idêntica ao meio MD, porém, a glicose foi substituída por $50 \mathrm{~g} \mathrm{~L}^{-1}$ de bagaço de cana-de-açúcar (Cardoso et al., 2018). Todos os cultivos foram realizados em frascos Erlenmeyer ( $250 \mathrm{~mL}$ ) contendo $60 \mathrm{~mL}$ de meio líquido. Os meios de cultivo foram autoclavados à $121^{\circ} \mathrm{C}$ por 20 min e, após a inoculação, os frascos foram mantidos em estufa incubadora BOD (Biochemical Oxygen Demand) a $28 \pm 1{ }^{\circ} \mathrm{C}$, na ausência de luz, sem agitação por 12 dias. Ao final do período de cultivo, o micélio foi separado do meio líquido por filtração à vácuo em papel filtro (Whatman $\mathrm{n}^{\circ}$. 1) e o filtrado, considerado extrato enzimático, foi usado para determinação das atividades enzimáticas. A biomassa micelial foi determinada por análise termogravimétrica acompanhando-se a massa durante secagem em estufa com circulação de ar a $60{ }^{\circ} \mathrm{C}$ até massa constante (Valle et al., 2014). Em virtude da característica particulada do meio BC a biomassa micelial produzida neste meio de cultivo não foi determinada.

\subsection{Ensaios enzimáticos}

\subsubsection{Atividade de celulase}

A atividade celulolítica dos extratos enzimáticos foi avaliada pelo método 3,5-DNS (ácido-3,5-dinitrosalicílico) (Miller, 1959) para determinação de açúcares redutores liberados de substratos celulósicos (Ghose, 1987).

A atividade de celulase total (ou FPase) foi determinada usando papel filtro Whatman $\mathrm{n}^{\circ} 1(1 \times 6 \mathrm{~cm}, \cong 50 \mathrm{mg})$ como substrato em reação de volume total $1,5 \mathrm{~mL}$ contendo: papel filtro, $1 \mathrm{~mL}$ de tampão citrato de sódio $(50 \mathrm{mM}, \mathrm{pH} 4,8)$ e $0,5 \mathrm{~mL}$ de extrato enzimático. A mistura reacional foi incubada a $50{ }^{\circ} \mathrm{C}$ por $60 \mathrm{~min}$. A atividade de endoglicanase (ou CMCase) foi determinada utilizando como substrato a carboximetilcelulose (CMC) $2 \%\left(\mathrm{~m} \mathrm{v}^{-1}\right)$ em tampão citrato de sódio $50 \mathrm{mM} \mathrm{pH} \mathrm{4,8.} \mathrm{A}$ reação de volume total $1 \mathrm{~mL}$ continha $0,5 \mathrm{~mL}$ de extrato enzimático e $0,5 \mathrm{~mL}$ de CMC $2 \%$ e foi incubada a $50{ }^{\circ} \mathrm{C}$ por 30 min. Após a incubação, adicionou-se $1 \mathrm{~mL}$ de 3,5-DNS à reação que foram submetidas à fervura $\left(100{ }^{\circ} \mathrm{C}\right)$ por 5 min. Após resfriamento rápido em banho de gelo, a liberação de açúcares redutores foi acompanhada por espectrofotometria a $540 \mathrm{~nm}$. Em todas as análises foram empregados controles analíticos, um contendo substrato na ausência de extrato enzimático e outro contendo o extrato enzimático na ausência de substrato. A concentração de açúcares foi determinada com base em uma curva padrão construída com concentrações conhecidas de glicose. Uma unidade (U) de atividade enzimática foi definida como a quantidade de enzima necessária para liberar $1 \mu \mathrm{mol}$ de glicose por minuto. 


\subsubsection{Atividade de lacase}

Uma solução (1 mM) de ABTS (2,2'-azino-bis (3-etilbenzotiazolina-6-sulfonato) (Valle et al., 2015) foi usada como substrato para determinação da atividade de lacase. As misturas reacionais foram compostas por $0,2 \mathrm{~mL}$ do extrato enzimático, $0,7 \mathrm{~mL}$ de água, $0,45 \mathrm{~mL}$ de tampão acetato de sódio $(0,1 \mathrm{M}, \mathrm{pH} 5,0)$ e $0,15 \mathrm{~mL}$ de ABTS. A mistura foi mantida a $30^{\circ} \mathrm{C}$ por 10 min e a taxa de oxidação do ABTS foi acompanhada por espectrofotometria a $420 \mathrm{~nm}\left(\varepsilon=36000 \mathrm{M}^{-1} \mathrm{~cm}^{-1}\right)$. Misturas contendo os mesmos componentes da reação, exceto o extrato; e mistura contendo os mesmos componentes, exceto o ABTS, foram utilizados como controles analíticos. Uma unidade (U) de atividade enzimática foi definida como a quantidade de enzima necessária para oxidar $1 \mu \mathrm{mol}$ de ABTS por minuto.

\subsubsection{Atividade de xilanase}

A atividade de xilanase foi determinada utilizando como substrato a xilana (birchwood 1\% $\mathrm{m} \mathrm{v}^{-1}$ ) (Bailey et al., 1992). A mistura reacional continha $1,8 \mathrm{ml}$ de xilana e $200 \mathrm{uL}$ do extrato enzimático. A mistura foi incubada a $50{ }^{\circ} \mathrm{C}$ por $5 \mathrm{~min}$. Após a incubação adicionou-se $1 \mathrm{~mL}$ de 3,5-DNS às reações que foram submetidas à fervura $\left(100{ }^{\circ} \mathrm{C}\right)$ por 15 min. Após resfriamento rápido em banho de gelo, a liberação de xilose foi acompanhada por espectrofotometria a $540 \mathrm{~nm}$. Em todas as análises foram empregados controles analíticos, um contendo xilana na ausência de extrato enzimático e um contendo o extrato enzimático na ausência de xilana. A concentração de xilose foi determinada com base em uma curva padrão construída com concentrações conhecidas de xilose. Uma unidade (U) de atividade enzimática foi definida como a quantidade de enzima necessária para liberar $1 \mu \mathrm{mol}$ de xilose por minuto.

\subsection{Análise estatística}

Em todas as etapas empregou-se metodologia quantitativa (Pereira et al., 2018). Todos os ensaios seguiram o delineamento inteiramente casualizado conduzidos em três repetições. Os resultados foram submetidos à análise de variância (ANOVA) e as diferenças significativas entre as médias e desvio padrão determinadas pelo teste de Scott-Knott a 5\% de probabilidade.

\section{Resultados e Discussão}

Todos os meios de cultivo avaliados promoveram o crescimento de Pleurotus spp., contudo, todas as espécies e cepas produziram mais biomassa micelial $(p \leq 0,05)$ no meio EM do que no meio MD (Tabela 1). As cepas de $P$. djamor foram as que mais cresceram no meio EM, seguidas de $P$. eryngii e $P$. pulmonarius. No meio MD, P. pulmonarius apresentou o melhor desempenho, seguido de $P$. ostreatus e $P$. djamor U16-25. As maiores diferenças de crescimento entre o meio EM e MD foram observadas para $P$. djamor U16-20 e $P$. citrinopileatus que produziram 6,6 e 5,5 vezes, respectivamente, mais biomassa no meio EM. Já a menor diferença de crescimento entre o meio EM e MD foi de $P$. ostreatus com uma diferença de menos de duas vezes. 
Tabela 1. Efeito do meio de cultivo na produção de biomassa micelial e na taxa de crescimento micelial de Pleurotus spp. crescidos em meio líquido extrato de malte $20 \mathrm{~g} \mathrm{~L}^{-1}(\mathrm{EM})$ e em meio definido com glicose $10 \mathrm{~g} \mathrm{~L}^{-1}$ (MD) por 12 dias.

\begin{tabular}{lcc|cc}
\hline \multirow{2}{*}{ Linhagens } & \multicolumn{2}{c|}{$\begin{array}{c}\text { Biomassa micelial } \\
\left(\mathbf{g ~ L}^{-1}\right)\end{array}$} & \multicolumn{2}{c}{$\begin{array}{c}\text { Taxa de crescimento micelial }(\mathbf{g} \\
\left.\mathbf{L}^{-1} \mathbf{d i a}^{-1}\right)\end{array}$} \\
\cline { 2 - 5 } & EM & MD & EM & MD $^{\text {EM }}$ \\
\hline Pleurotus citrinopileatus U16-23 & $5,8 \pm 0,4^{\mathrm{cA}}$ & $1,0 \pm 0,0^{\mathrm{fB}}$ & $0,5 \pm 0,03^{\mathrm{cA}}$ & $0,1 \pm 0,00^{\mathrm{fB}}$ \\
Pleurotus djamor U16-20 & $7,1 \pm 0,6^{\mathrm{aA}}$ & $1,1 \pm 0,2^{\mathrm{fB}}$ & $0,6 \pm 0,05^{\mathrm{aA}}$ & $0,1 \pm 0,02^{\mathrm{fB}}$ \\
Pleurotus djamor U16-25 & $7,5 \pm 0,1^{\mathrm{aA}}$ & $2,7 \pm 0,4^{\mathrm{cB}}$ & $0,6 \pm 0,09^{\mathrm{aA}}$ & $0,2 \pm 0,03^{\mathrm{cB}}$ \\
Pleurotus djamor U16-28 & $7,0 \pm 0,2^{\mathrm{aA}}$ & $1,6 \pm 0,3^{\mathrm{eB}}$ & $0,6 \pm 0,02^{\mathrm{aA}}$ & $0,1 \pm 0,02^{\mathrm{eB}}$ \\
Pleurotus eryngii U16-30 & $6,5 \pm 0,1^{\mathrm{bA}}$ & $2,2 \pm 0,0^{\mathrm{dB}}$ & $0,5 \pm 0,00^{\mathrm{bA}}$ & $0,2 \pm 0,00^{\mathrm{dB}}$ \\
Pleurotus ostreatus U16-22 & $5,0 \pm 0,3^{\mathrm{dA}}$ & $3,2 \pm 0,1^{\mathrm{bB}}$ & $0,4 \pm 0,03^{\mathrm{dA}}$ & $0,3 \pm 0,00^{\mathrm{bB}}$ \\
Pleurotus pulmonarius $\mathrm{U} 16-21$ & $6,4 \pm 0,0^{\mathrm{bA}}$ & $3,6 \pm 0,0^{\mathrm{aB}}$ & $0,5 \pm 0,00^{\mathrm{bA}}$ & $0,3 \pm 0,00^{\mathrm{aB}}$ \\
\hline
\end{tabular}

Os resultados são expressos como média \pm desvio padrão $(n=3)$. Letras minúsculas na mesma coluna comparam diferentes linhagens e letras maiúsculas na mesma linha comparam os meios EM e MD. Médias indicadas por letras diferentes apresentam diferença estatística de acordo com o teste de Skott-Knott $(p \leq 0,05)$. Fonte: Autores.

O extrato de malte contém uma mistura de monossacarídeos, oligossacarídeos e polissacarídeos, resultantes da hidrólise enzimática de cereais maltados (Paik et al., 1991). Esta mistura de açúcares inclui açúcares de cadeia longa, glicose, frutose, sacarose e principalmente maltose $(50-65 \%)$. Nossos resultados demonstram que as diferentes espécies de Pleurotus avaliadas cresceram mais no EM do que no meio MD com glicose como única fonte de carbono. Apesar de a glicose e outros monossacarídeos usualmente estimularem o crescimento micelial e o acúmulo de biomassa de Pleurotus (Bellettini et al., 2019), outros açúcares podem garantir um crescimento significativo. Pleurotus florida cultivado em meio líquido com diferentes mono e oligossacarídeos produziu maior rendimento de biomassa micelial com glicose e frutose, porém, o acúmulo de biomassa no meio com maltose também foi expressivo (Gbolagade et al., 2006). Os mesmos autores relataram que o cultivo de $P$. florida em extrato de malte produziu 33\% mais biomassa que no meio com celulose. O cultivo submerso do micélio de $P$. albidus gerou mais biomassa nos meios com sacarose e maltose, sem diferença significativa entre eles, do que no meio com glicose corroborando nossas observações (Kirsch et al., 2016). Monossacarídeos são prontamente metabolizáveis e, apesar de requererem a ação de enzimas para hidrólise inicial, os oligossacarídeos como a maltose e até mesmo polissacarídeos podem dar suporte para o crescimento micelial (Rathore et al., 2019).

Os resultados da atividade enzimática de Pleurotus spp. mostraram que a produção das diferentes enzimas ocorreu de forma dependente das espécies de Pleurotus e os fungos exibiram diferentes respostas aos meios de cultivo avaliados. Os resultados da atividade de lacase encontram-se na Figura 1 e indicam que o tipo de meio de cultivo afetou a atividade de lacase das cepas de Pleurotus. Todas as linhagens produziram lacase em todos os meios de cultivo testados. Quatro das sete cepas avaliadas apresentaram maior atividade de lacase quando cultivadas no meio MD e as maiores atividades neste meio foram de P. djamor U16-25 (22243 $\left.\pm 745 \mathrm{U} \mathrm{L}^{-1}\right)$ e $P$. pulmonarius $\left(20924 \pm 46 \mathrm{U} \mathrm{L}^{-1}\right)$. Vale destacar que, apesar de o micélio ter se desenvolvido menos no meio MD (Tabela 1), a produção de lacase foi maior nesta condição de cultivo, refletindo mudanças metabólicas e/ou na expressão de genes de lacase.

As sete linhagens avaliadas produziram lacase no meio BC, contudo, apenas $P$. citrinopileatus $\left(7700 \pm 563 \mathrm{U} \mathrm{L}^{-1}\right)$ e $P$. djamor U16-20 (9620 $\left.\pm 388 \mathrm{U} \mathrm{L}^{-1}\right)$ produziram mais lacase no meio BC que nos demais meios. De modo geral, o cultivo no meio EM foi o que apresentou as menores atividades de lacase. Apenas a linhagem $P$. djamor U16-25 produziu atividade de lacase $\left(16630 \pm 2974 \mathrm{U} \mathrm{L}^{-1}\right)$ expressiva no meio EM, contudo esta foi a cepa que mais produziu lacase em todos os meios de 
cultivo. As diferentes cepas de $P$. djamor apresentaram atividades de lacase discrepantes nos meios avaliados. A linhagem de $P$. djamor com a maior $(p \leq 0,05)$ atividade de lacase no meio de cultivo MD foi U16-25, com atividade três vezes e cinco vezes maior do que as cepas U16-28 e U16-20, respectivamente, no mesmo meio. P. djamor U16-20 e U16-28, por outro lado, produziram maior atividade de lacase no meio BC. Porém, a atividade de lacase de U16-28 não foi diferente $(p>0,05)$ nos meios BC e MD. P. djamor U16-20 foi a única cepa que produziu menor atividade de lacase no meio MD. Os resultados de atividade de lacase observados para as diferentes cepas de $P$. djamor evidenciam diferenças nas características biotecnológicas da espécie e indicam que a variabilidade de respostas de Pleurotus spp. ao meio de cultivo ocorre de forma interespecífica e intraespecífica.

Figura 1. Atividade de lacase de Pleurotus spp. cultivados em extrato de malte $2 \%$ (EM), meio definido com glicose $10 \mathrm{~g} \mathrm{~L}^{-1}$ (MD) ou em meio com bagaço de cana-de-açúcar $50 \mathrm{~g} \mathrm{~L}^{-1}$ (BC) por 12 dias.

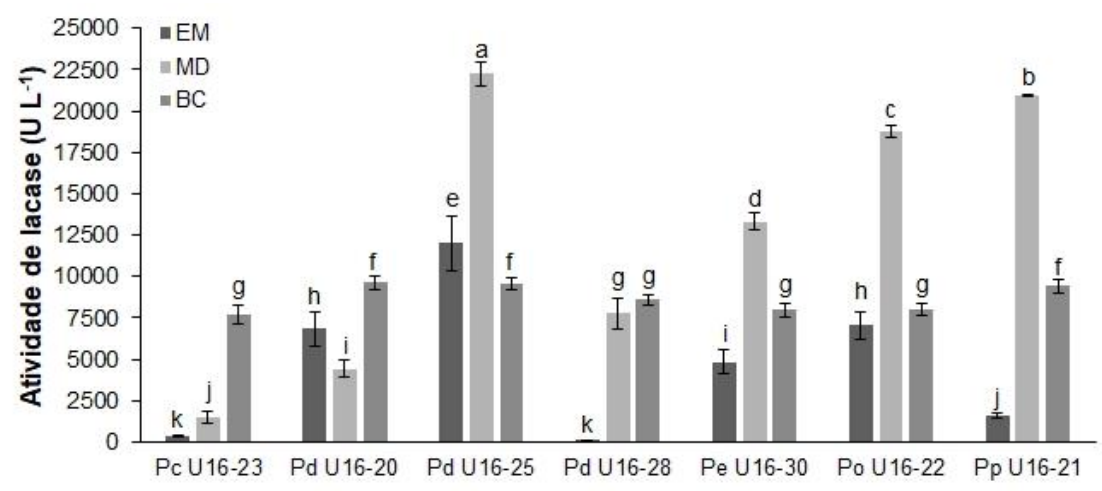

Os resultados são expressos como média \pm desvio padrão $(\mathrm{n}=3)$. Pc - Pleurotus citrinopileatus, $\mathrm{Pd}-$ Pleurotus djamor, Pe - Pleurotus eryngii, Po - Pleurotus ostreatus, Pp - Pleurotus pulmonarius. Médias indicadas por letras diferentes apresentam diferença estatística de acordo com o teste de Skott-Knott $(p \leq 0,05)$. Fonte: Autores.

A fonte e a concentração de carbono são determinantes na atividade de lacase de diferentes espécies de fungos (Shafiq et al., 2016). Segundo Majeau et al. (2010), fontes de carbono facilmente metabolizáveis como glicose, manitol e celobiose podem favorecer maior atividade de lacase do que fontes lentamente assimiláveis como celulose e material lignocelulósico. Para as linhagens avaliadas, o meio MD que continha glicose em quantidade limitada $\left(10 \mathrm{~g} \mathrm{~L}^{-1}\right)$ favoreceu a produção de lacase e o meio EM rico em açúcares redutores ( $60 \%)$, promoveu atividade bem menor, indicando que maiores concentrações de açúcares podem reduzir a produção de lacase de Pleurotus spp. (Federici et al., 2009). Hou et al. (2004), verificaram que $P$. ostreatus produziu mais lacase quando cultivado com fontes de carbono facilmente assimiláveis (glicose $-95 \mathrm{U} \mathrm{mL}^{-1} \mathrm{e}$ celulose $-60 \mathrm{U} \mathrm{mL}^{-1}$ ). Entretanto, para muitas espécies de fungos a glicose é um repressor da produção de lacase e a concentração de açúcares no meio de cultivo afeta diretamente a expressão gênica desta enzima. O meio BC foi o segundo melhor para a produção de lacase das cepas de Pleurotus. O bagaço de cana-de-açúcar é rico em lignina (18-25\%) e açúcares complexos como a celulose (41-50\%) e hemicelulose (15-25\%), que podem ser usados como fonte de carbono pelo fungo (Ferreira et al., 2010). O bagaço também contém polifenóis solúveis (50 g GAE/100 g extrato) (Federici et al., 2009) que durante o cultivo submerso podem atuar como indutores naturais de lacase (Cardoso et al., 2018). A produção de lacase de Pleurotus spp. no meio BC se mostrou promissora e poderá ser otimizada analisando-se outros componentes do meio como a fonte e a concentração de nitrogênio, minerais e indutores enzimáticos (Souza et al., 2020).

Os resultados das atividades celulolíticas encontram-se na Tabela 2. No meio BC não se detectou atividades celulolíticas de nenhuma das cepas e a produção destas enzimas nos meios EM e MD também foi limitada. Para as cepas $P$. 
citrinopileatus e $P$. djamor U16-25 não se detectou atividade de celulases em nenhum dos meios de cultivo. Pleurotus ostreatus e $P$. pulmonarius apresentaram atividade de celulase apenas no meio EM, enquanto $P$. djamor U16-28 produziu celulases apenas no meio MD. Por outro lado, $P$. djamor U16-20 e $P$. eryngii produziram atividade de celulase tanto no meio EM quanto no meio MD e foram as cepas com as maiores atividades enzimáticas (endoglicanase no meio EM). De modo geral, as maiores atividades de celulase ocorreram no meio EM. Os resultados sugerem que há grande variação na fisiologia das espécies de Pleurotus que são evidenciadas dependendo do meio de cultivo.

Tabela 2. Atividade de celulases de Pleurotus spp. cultivados em extrato de malte 2\% (EM), meio definido com glicose $10 \mathrm{~g}$ $\mathrm{L}^{-1}(\mathrm{MD})$ ou em meio com bagaço de cana-de-açúcar $50 \mathrm{~g} \mathrm{~L}^{-1}(\mathrm{BC})$ por 12 dias.

\begin{tabular}{|c|c|c|c|c|c|c|}
\hline \multirow[t]{2}{*}{ Linhagens } & \multicolumn{3}{|c|}{$\begin{array}{l}\text { Atividade de celulase total } \\
\qquad\left(\mathbf{U ~ L ~ L ~}^{-1}\right)\end{array}$} & \multicolumn{3}{|c|}{$\begin{array}{c}\text { Atividade de endoglicanase } \\
\left(\mathbf{U}_{\left.\mathbf{L}^{-1}\right)}\right.\end{array}$} \\
\hline & EM & MD & $\mathbf{B C}$ & EM & MD & BC \\
\hline Pleurotus citrinopileatus U16-23 & nd & nd & nd & nd & nd & nd \\
\hline Pleurotus djamor U16-20 & nd & $246 \pm 13^{b}$ & nd & $1563 \pm 57^{\mathrm{aA}}$ & $805 \pm 95^{\mathrm{aB}}$ & nd \\
\hline Pleurotus djamor U16-25 & nd & nd & nd & nd & nd & nd \\
\hline Pleurotus djamor U16-28 & nd & $393 \pm 34^{\mathrm{a}}$ & nd & nd & $471 \pm 56^{b}$ & nd \\
\hline Pleurotus eryngii U16-30 & nd & $136 \pm 17^{\mathrm{c}}$ & nd & $1673 \pm 17^{\mathrm{aA}}$ & $76 \pm 9^{\mathrm{cB}}$ & nd \\
\hline Pleurotus ostreatus U16-22 & $303 \pm 96^{\mathrm{a}}$ & nd & nd & $1263 \pm 41^{b}$ & nd & nd \\
\hline Pleurotus pulmonarius U16-21 & nd & nd & nd & $1227 \pm 224^{b}$ & nd & nd \\
\hline
\end{tabular}

Os resultados são expressos como média \pm desvio padrão $(n=3)$. Letras minúsculas na mesma coluna comparam diferentes linhagens e letras maiúsculas na mesma linha comparam os meios EM e MD. Médias indicadas por letras diferentes apresentam diferença estatística de acordo com o teste de Skott-Knott $(p \leq 0,05)$. nd = não detectado. Fonte: Autores.

Fatores como composição do meio como fonte e concentração de carbono e nitrogênio; modo de cultivo; e espécie fúngicas interferem grandemente na produção de celulases por fungos da podridão branca como Pleurotus spp. (Okal et al., 2020). Portanto, as condições ideais para produção dessas enzimas variam entre espécies e entre linhagens e deverão ser avaliadas e padronizadas dependendo do objetivo da produção. A fonte de carbono é importante para a atividade enzimática e em Pleurotus a maior disponibilidade de açúcares prontamente metabolizáveis como no meio EM favoreceu a produção de endoglicanase. Nossos resultados são similares aos relatados por Goyal \& Soni (2011) que relataram que as atividades de celulase total e endoglicanase de $P$. florida foram significativas quando o meio de cultivo líquido contento $\mathrm{CMC}\left(10 \mathrm{~g} \mathrm{~L}^{-1}\right)$ foi suplementado com $0,5 \%$ de extrato de malte. Nossos resultados, no entanto, foram superiores aos relatados no referido estudo. Pleurotus eryngii no meio EM produziu 3,6 vezes mais endoglicanase que $P$. florida (406 U L-1) e P. ostreatus produziu 3 vezes mais celulase total no meio EM que P. florida $\left(101 \mathrm{U} \mathrm{L}^{-1}\right)$ (Goyal e Soni, 2011). Debnath et al. (2019), por outro lado, ao cultivarem $P$. giganteus recém isolado em meio de cultivo contendo CMC $\left(10 \mathrm{~g} \mathrm{~L}^{-1}\right)$ relatou atividade de endoglicanase superior a $6000 \mathrm{U} \mathrm{L}^{-1}$.

Os resultados da atividade de xilanase de Pleurotus spp. nos diferentes meios de cultivos estão na Figura 2. Houve produção de xilanase em todos os meios de cultivo, porém, as maiores atividades de todas as cepas foram observadas no meio EM seguido do meio MD e as menores atividade de xilanase foram no meio BC. No meio EM as cepas de $P$. djamor U16-28 (67154 $\left.\pm 1597 \mathrm{U} \mathrm{L}^{-1}\right)$ e U16-25 (64180 $\left.\pm 2846 \mathrm{U} \mathrm{L}^{-1}\right)$ produziram as maiores atividades de xilanase. Na mesma condição de cultivo, P. citrinopileatus também produziu elevada atividade de xilanase $\left(60213 \pm 2480 \mathrm{U} \mathrm{L}^{-1}\right)$. A atividade de xilanase de todas as cepas de Pleurotus foi menor no meio MD do que no meio EM, com diferenças que variaram de $24 \%$ para $P$. pulmonarius a $57 \%$ para $P$. djamor U16-25. No meio BC a maior atividade de xilanase foi de $P$. citrinopileatus $(9944 \pm 216 \mathrm{U}$ $\left.\mathrm{L}^{-1}\right)$ e as demais cepas produziram em média $2380 \pm 540 \mathrm{U} \mathrm{L}^{-1}$ de xilanase, sem diferença entre elas. 
Figura 2. Atividade de xilanase de Pleurotus spp. cultivados em extrato de malte $2 \%$ (EM), meio definido com glicose $10 \mathrm{~g} \mathrm{~L}$ ${ }^{1}$ (MD) ou em meio com bagaço de cana-de-açúcar $50 \mathrm{~g} \mathrm{~L}^{-1}$ (BC) por 12 dias.

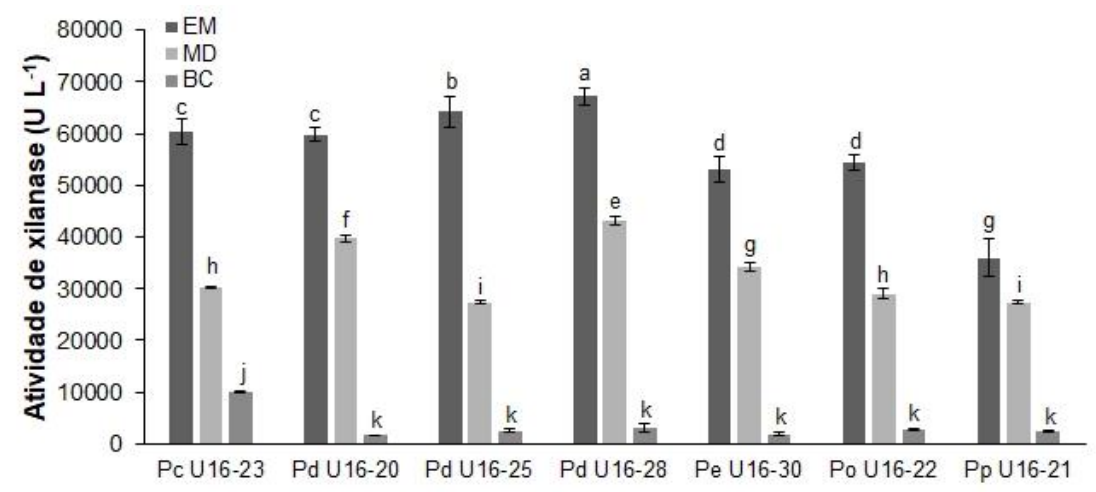

Os resultados são expressos como média \pm desvio padrão $(\mathrm{n}=3)$. Pc - Pleurotus citrinopileatus, $\mathrm{Pd}-$ Pleurotus djamor, Pe - Pleurotus eryngii, Po - Pleurotus ostreatus, Pp - Pleurotus pulmonarius. Médias indicadas por letras diferentes apresentam diferença estatística de acordo com o teste de Skott-Knott $(p \leq 0,05)$. Fonte: Autores.

Fungos produzem xilanases que podem ser constitutivas ou induzíveis (Bhardwaj et al., 2019). Dessa forma, acreditase que a maioria dos fungos produzam certa quantidade de enzima em nível basal e que as condições do meio de cultivo, particularmente a presença de oligossacarídeos derivados da hemicelulose, induzam a atividade de xilanase. HernándezDomínguez et al. (2017) cultivaram P. ostreatus em meio líquido contendo glicose $\left(10 \mathrm{~g} \mathrm{~L}^{-1}\right)$ ou glicose e indutores (CMC ou xilana) e verificaram maior atividade de xilanase $\left(\sim 1500 \mathrm{U} \mathrm{L} \mathrm{L}^{-1}\right)$ no meio com glicose e indutores. Nossos resultados foram superiores em todos os meios de cultivo. P. ostreatus, por exemplo, produziu 36 vezes mais xilanase no meio EM, 19 vezes mais xilanase no meio MD e 2 vezes mais no meio BC.

Xilanases são de grande importância na indústria de papel e celulose, pois seu uso na hidrólise de xilana facilita a liberação de lignina durante o processo de polpação reduzindo o uso de compostos clorados no branqueamento da polpa celulósica (Hutterer et al., 2017). Contudo, a presença de celulases nas formulações a base de xilanase podem comprometer o processo polpação danificando as fibras celulósicas o que pode gerar um papel de qualidade inferior (Subramaniyan \& Prema, 2000). Dessa forma, este setor industrial busca por xilanases livres de celulases para que haja a degradação seletiva da hemicelulose com dano mínimo à celulose. As linhagens avaliadas neste estudo podem ser interessantes na produção de xilanases livres de celulases. Pleurotus citrinopileatus e $P$. djamor U16-25, por exemplo, não produziram celulases em níveis detectáveis nos meios de cultivo avaliados, porém, produziram excelente atividade de xilanase, principalmente no meio EM com atividades superiores a $60000 \mathrm{U} \mathrm{L}^{-1}$. Da mesma forma, P. ostreatus e P. pulmonarius quando cultivados em meio MD produzem apenas xilanase, ambos com atividades superiores a $27000 \mathrm{U} \mathrm{L}^{-1}$. Adicionalmente, linhagens como P. djamor U1625 apresentam características ideais para uso em polpação de celulose uma vez que esta cepa produz uma combinação de elevadas atividades de lacase e xilanase e pouca ou nenhuma atividade de celulase.

\section{Conclusão}

A atividade enzimática e a produção de biomassa micelial de Pleurotus spp. são diferentes nos meios de cultivo avaliados e variam de forma dependente da espécie. O meio extrato de malte favorece a produção de biomassa micelial de todas as linhagens. Todas as cepas de Pleurotus spp. produzem lacase e as maiores atividades ocorrem quando são cultivadas em meio definido com glicose. De modo geral, as maiores atividades de celulase ocorrem no meio extrato de malte. Todas as linhagens produziram elevada atividade de xilanase nos meios extrato de malte e definido. Pleurotus djamor U16-25 se destaca 
como eficiente produtora de lacase e xilanase livre de celulase mostrando potencial para aplicação na indústria de papel e celulose. Nossos resultados demonstram que as cepas de Pleurotus spp. avaliadas neste estudo têm grande potencial para despolimerização de matéria orgânica com elevadas concentrações de hemicelulose e lignina. Novos estudos devem ser realizados para avaliar outras condições do meio de cultivo, além de estudos de cinética e estabilidade enzimática.

\section{Agradecimentos}

Os autores agradecem à Universidade Paranaense, Programa de Pós-graduação em Biotecnologia Aplicada à Agricultura, Instituto Federal do Paraná, Coordenação de Aperfeiçoamento de Pessoal de Nível Superior (CAPES - código financeiro 001), Conselho Nacional de Desenvolvimento Científico e Tecnológico (CNPq, 307953/2017-3) e Fundação Araucária pelo apoio financeiro e bolsas. Agradecem Mycelia Mushroom Spawn Company da Bélgica e Instituto de Ecologia A. C. (INECOL) do México pela doação de cepas de fungos.

\section{Referências}

Anugraha, S., Swaminathan, T., Swaminathan, D., Meyyappan, N., \& Parthiban, R. (2016). Enzymes in Platform Chemical Biorefinery. In: Brar, S.K.; Sarma, S.J.; Pakshirajan, K. (Eds.) Platform Chemical Biorefinery. Elsevier, 451-469.

Bailey, M. J., Biely, P., \& Poutanen, K. (1992). Interlaboratory testing of methods for assay of xylanase activity. Journal of Biotechnology, 23(3), 257-270.

Barcelos, M. C., Ramos, C. L., Kuddus, M., Rodriguez-Couto, S., Srivastava, N., Ramteke, P. W., Mishra, P. K., \& Molina, G. (2020). Enzymatic potential for the valorization of agro-industrial by-products. Biotechnology Letters, 42, 1799-1827.

BCC Research. Global markets for enzymes in industrial applications. (2018). https://www.bccresearch.com/market-research/biotechnology/global-marketsfor-enzymes-in-industrial-applications.html.

Bellettini, M. B., Fiorda, F. A., Maieves, H. A., Teixeira, G. L., Ávila, S., Hornung, P. S., Maccari Jr, A., \& Ribani, R. H. (2019). Factors affecting mushroom Pleurotus spp. Saudi Journal of Biological Sciences, 26(4), 633-646.

Bettin, F., Cousseau, F., Martins, K., Boff, N. A., Zaccaria, S., da Silveira, M. M., \& Dillon, A. J. P. (2019). Phenol removal by laccases and other phenol oxidases of Pleurotus sajor-caju PS-2001 in submerged cultivations and aqueous mixtures. Journal of Environmental Management, 236 , 581-590.

Bhardwaj, N., Kumar, B., \& Verma, P. (2019). A detailed overview of xylanases: an emerging biomolecule for current and future prospective. Bioresources and Bioprocessing, 6(1), 40.

Cardoso, B. K., Linde, G. A., Colauto, N. B., \& Valle, J. S. (2018). Panus strigellus laccase decolorizes anthraquinone, azo, and triphenylmethane dyes. Biocatalysis and Agricultural Biotechnology, 16, 558-563.

Debnath, G., Das, P., \& Saha, A. K. (2020). Screening and characterization of extracellular cellulase enzyme produced by wild edible mushroom Pleurotus giganteus. Indian Journal of Natural Products and Resources, 10(3), 195-199.

Elisashvili, V., Kachlishvili, E., Asatiani, M. D., Darlington, R., \& Kucharzyk, K. H. (2017). Physiological peculiarities of lignin-modifying enzyme production by the white-rot basidiomycete Coriolopsis gallica strain BCC 142. Microorganisms, 5(4), 73.

Faria, M. G., Valle, J. S., Lopes, A. D., Junior, A. C. G., Dragunski, D. C., Colauto, N. B., \& Linde, G. A. (2018). Mycelial bioaccumulation of lithium $\left(\mathrm{Li}_{2} \mathrm{CO}_{3}\right)$ in Pleurotus ostreatus. International Journal of Medicinal Mushrooms, 20(9):901-907.

Federici, F., Fava, F., Kalogerakis, N., \& Mantzavinos, D. (2009). Valorisation of agro-industrial by-products, effluents and waste: concept, opportunities and the case of olive mill wastewaters. Journal of Chemical Technology \& Biotechnology, 84(6), 895-900.

Fernandes, Â., Barros, L., Martins, A., Herbert, P., \& Ferreira, I. C. (2015). Nutritional characterisation of Pleurotus ostreatus (Jacq. ex Fr.) P. Kumm. produced using paper scraps as substrate. Food Chemistry, 169, 396-400.

Ferreira, L. F., Aguiar, M., Pompeu, G., Messias, T. G., \& Monteiro, R. R. (2010). Selection of vinasse degrading microorganisms. World Journal of Microbiology and Biotechnology, 26(9), 1613-1621.

Gbolagade, J., Sobowale, A., \& Adejoye, D. (2006). Optimization of sub-merged culture conditions for biomass production in Pleurotus florida (mont.) Singer, a Nigerian edible fungus. African Journal of Biotechnology, 5(16), 1464-1469. 
Ghose, T. K. (1987). Measurement of cellulase activities. Pure and Applied Chemistry, 59(2), 257-268.

Goyal, M., \& Soni, G. (2011). Production and characterization of cellulolytic enzymes by Pleurotus florida. African Journal of Microbiology Research, 5(10), $1131-1136$

Grimm, D., \& Wösten, H. A. (2018). Mushroom cultivation in the circular economy. Applied Microbiology and Biotechnology, 102(18), 7795-7803.

Hasmann, F. A., Vieira Cortez, D., Pessoa Júnior, A., \& Conceição Roberto, I. (2003). Optimization of beta-xylosidase recovery by reversed micelles using response surface methodology. Electronic Journal of Biotechnology, 6(2), 153-160.

Hernández-Domínguez, E. M., Sánchez, C., \& Díaz-Godínez, G. (2017). Production of laccases, cellulases and xylanases of Pleurotus ostreatus grown in liquid-state fermentation. Mexican Journal of Biotechnology, 2(2), 169-176.

Hou, H., Zhou, J., Wang, J., Du, C., \& Yan, B. (2004). Enhancement of laccase production by Pleurotus ostreatus and its use for the decolorization of anthraquinone dye. Process Biochemistry, 39(11), 1415-1419.

Hutterer, C., Kliba, G., Punz, M., Fackler, K., \& Potthast, A. (2017). Enzymatic pulp upgrade for producing high-value cellulose out of a Kraft paper pulp. Enzyme and Microbial Technology, 102, 67-73.

Jové, P., Olivella, M. À., Camarero, S., Caixach, J., Planas, C., Cano, L., \& de las Heras, F. X. (2016). Fungal biodegradation of anthracene-polluted cork: A comparative study. Journal of Environmental Science and Health, 51(1), 70-77.

Kirsch, L. D. S., Macedo, A. J. P. D., \& Teixeira, M. F. S. (2016). Production of mycelial biomass by the Amazonian edible mushroom Pleurotus albidus. Brazilian Journal of Microbiology, 47(3), 658-664.

Knop, D., Yarden, O., \& Hadar, Y. (2015). The ligninolytic peroxidases in the genus Pleurotus: divergence in activities, expression, and potential applications. Applied Microbiology and Biotechnology, 99(3), 1025-1038.

Kunjadia, P. D., Sanghvi, G. V., Kunjadia, A. P., Mukhopadhyay, P. N., \& Dave, G. S. (2016). Role of ligninolytic enzymes of white rot fungi (Pleurotus spp.) grown with azo dyes. SpringerPlus, 5(1), 1487.

Majeau, J. A., Brar, S. K., \& Tyagi, R. D. (2010). Laccases for removal of recalcitrant and emerging pollutants. Bioresource Technology, 101(7), 2331-2350.

Menezes, C. R., Silva, Í. S., Pavarina, É. C., Dias, E. F. G., Dias, F. G., Grossman, M. J., \& Durrant, L. R. (2009). Production of xylooligosaccharides from enzymatic hydrolysis of xylan by the white-rot fungi Pleurotus. International Biodeterioration \& Biodegradation, 63(6), 673-678.

Miller, G. L. (1959). Use of dinitrosalicylic acid reagent for determination of reducing sugar. Analytical Chemistry, 31(3), 426-428.

Mishra, K. K., Pal, R. S., Arunkumar, R., Chandrashekara, C., Jain, S. K., \& Bhatt, J. C. (2013). Antioxidant properties of different edible mushroom species and increased bioconversion efficiency of Pleurotus eryngii using locally available casing materials. Food chemistry, 138(2-3), 1557-1563.

Moreno, A. D., Ibarra, D., Alvira, P., Tomás-Pejó, E., \& Ballesteros, M. (2015). A review of biological delignification and detoxification methods for lignocellulosic bioethanol production. Critical Reviews in Biotechnology, 35(3), 342-354.

Okal, E. J., Aslam, M. M., Karanja, J. K., \& Nyimbo, W. J. (2020). Mini review: Advances in understanding regulation of cellulase enzyme in white-rot basidiomycetes. Microbial Pathogenesis, 147, 104410.

Paik, J., Low, N. H., \& Ingledew, W. M. (1991). Malt extract: relationship of chemical composition to fermentability. Journal of the American Society of Brewing Chemists, 49(1), 8-13.

Pereira, A. S., Shitsuka, D. M., Parreira, F. B., \& Shitsuka, R. (2018). Metodologia da pesquisa científica [recurso eletrônico[eBook]. Santa Maria. Ed. UAB/NTE/UFSM. https://repositorio.ufsm.br/bitstream/handle/1/15824/Lic_Computa cao_MetodologiaPesquisa-Cientifica.pdf?sequence=1.

Rampinelli, J. R., Silveira, M. L. L., Gern, R. M. M., Furlan, S. A., Ninow, J. L., \& Wisbeck, E. (2010). Valor nutricional de Pleurotus djamor cultivado em palha de bananeira. Alimentos e Nutrição, 21(2), 197-202.

Rathore, H., Prasad, S., Kapri, M., Tiwari, A., \& Sharma, S. (2019). Medicinal importance of mushroom mycelium: Mechanisms and applications. Journal of Functional Foods, 56, 182-193.

Ravindran, R., Hassan, S. S., Williams, G. A., \& Jaiswal, A. K. (2018). A review on bioconversion of agro-industrial wastes to industrially important enzymes. Bioengineering, 5(4), 93.

Ritota, M., \& Manzi, P. (2019). Pleurotus spp. cultivation on different agri-food by-products: example of biotechnological application. Sustainability, 11(18), 5049 . 
Royse, D. J., Baars, J., \& Tan, Q. (2017). Current overview of mushroom production in the world. In: Cunha, Z.D., Pardo-Gimenez, A. (Eds.). Edible and Medicinal Mushrooms: technology and applications, John Wiley \& Sons, pp. 5-13.

Scheid, S. S., Faria, M. G. I., Velasquez, L. G., do Valle, J. S., Gonçalves, A. C., Dragunski, D. C., Colauto, N.B., \& Linde, G. A. (2020). Iron biofortification and availability in the mycelial biomass of edible and medicinal basidiomycetes cultivated in sugarcane molasses. Scientific Reports, $10(1), 1-6$.

Sekan, A. S., Myronycheva, O. S., Karlsson, O., Gryganskyi, A. P., \& Blume, Y. (2019). Green potential of Pleurotus spp. in biotechnology. PeerJ, 7, e6664.

Shafiq, A., Masood, F., Naseer, R., Naveed, S., Rasool, I. G., \& Rahman, A. (2016). Production, purification and characterization of laccase from white rot fungus. Pakistan Journal of Science, 68(3), 259-267.

Sharma, B., Dangi, A. K., \& Shukla, P. (2018). Contemporary enzyme based technologies for bioremediation: a review. Journal of Environmental Management, 210, 10-22.

Singh, S. K., Khajuria, R., \& Kaur, L. (2017). Biodegradation of ciprofloxacin by white rot fungus Pleurotus ostreatus. 3 Biotech, 7(1), 69.

Souza, G. P. N., Halabura, M. I. W., Avelino, K. V., Costa, M. R., Santana, T. T., Kassem, A. S. S., Marim, R. A., Nunes, M. G. I. F., Colauto, G. A., Colauto, N. B., \& Valle, J. S. (2020). Lacase de Agaricus subrufescens cultivado em meio com melaço de cana-de-açúcar promove a descoloração de corantes sintéticos. Research, Society and Development, 9(12), e12391210942.

Subramaniyan, S., \& Prema, P. (2000). Cellulase-free xylanases from Bacillus and other microorganisms. FEMS Microbiology Letters, $183(1), 1-7$.

Tavares, M. F., Avelino, K. V., Araújo, N. L., Marim, R. A., Linde, G. A., Colauto, N. B., \& do Valle, J. S. (2020). Decolorization of azo and anthraquinone dyes by crude laccase produced by Lentinus crinitus in solid state cultivation. Brazilian Journal of Microbiology, 51(1), 99-106.

Umeo, S. H., Souza, G. P. N., Rapachi, P. M., Garcia, D. M., Paccola-Meirelles, L. D., Valle, J. S., Colauto, N. B., \& Linde, G. A. (2015). Screening of basidiomycetes in submerged cultivation based on antioxidant activity. Genetics and Molecular Research, 14(3), 9907-9914.

Valle, J. S., Vandenberghe, L. P. S., Santana, T. T., Almeida, P. H., Pereira, A. M., Linde, G. A., Colauto, N.B., \& Soccol, C. R. (2014). Optimum conditions for inducing laccase production in Lentinus crinitus. Genetics and Molecular Research, 13(4), 8544-8551.

Valle, J. S., Vandenberghe, L. P. S., Oliveira, A. C. C., Tavares, M. F., Linde, G. A., Colauto, N. B., \& Soccol, C. R. (2015). Effect of different compounds on the induction of laccase production by Agaricus blazei. Genetics and Molecular Research, 14(4), 15882-15891.

Várnai, A., Siika-aho, M., \& Viikari, L. (2010). Restriction of the enzymatic hydrolysis of steam-pretreated spruce by lignin and hemicellulose. Enzyme and Microbial Technology, 46(3-4), 185-193.

Vetvicka, V., Gover, O., Karpovsky, M., Hayby, H., Danay, O., Ezov, N., \& Schwartz, B. (2019). Immune-modulating activities of glucans extracted from Pleurotus ostreatus and Pleurotus eryngii. Journal of Functional Foods, 54, 81-91.

Yin, C., Fan, X., Liu, C., Fan, Z., Shi, D., Yao, F., Cheng, F., \& Gao, H. (2019). The antioxidant properties, tyrosinase and $\alpha$-glucosidase inhibitory activities of phenolic compounds in different extracts from the golden oyster mushroom, Pleurotus citrinopileatus (Agaricomycetes). International Journal of Medicinal Mushrooms, 21(9).

Yokota, M. E., Frison, P. S., Marcante, R. C., Jorge, L. F., Valle, J. S., Dragunski, D. C., Colauto, N.B., \& Linde, G. A. (2016). Iron translocation in Pleurotus ostreatus basidiocarps: production, bioavailability, and antioxidant activity. Genetics and Molecular Research, 15(1), gmr.15017888.

Zaghi Junior, L. L., Bertéli, M. B. D., de Freitas, J. D. S., de Oliveira Filho, O. B. Q., Lopes, A. D., Ruiz, S. P., Valle, J.S., Linde, G.A. \& Colauto, N. B. (2020). Five-year cryopreservation at $-80^{\circ} \mathrm{C}$ of edible and medicinal basidiomycetes by wheat grain technique. Journal of Microbiological Methods, 176 , 106030 . 